\title{
Estimates for the composition of the carathéodory and homotopy operators
}

\section{Zhaoyang Tang ${ }^{*}$ and Jianmin Zhu}

\section{${ }^{*}$ Correspondence:}

tzymath@gmail.com

Department of Mathematics and

System Science, National University

of Defense Technology, Changsha,

P.R. China

\begin{abstract}
In the present paper, we deal with the composition of carathéodory and homotopy operators for differential forms satisfying the A-harmonic equation in the bounded and convex domain. We obtain estimates for the composition and the form of inequalities with weights. Moreover, we also obtain the composition for the gradient, carathéodory, and homotopy operators. Then we obtain the $W^{1, p}$ norm estimates for the composition operators.
\end{abstract}

Keywords: differential forms; composition operators; norm estimates

\section{Introduction}

The purpose of this paper is to establish the inequalities for the composition of the homotopy operator $T$ and the carathéodory operator $G$ applied to differential forms in $\mathbf{R}^{n}$, $n \geq 2$. The homotopy operator $T$ is widely used in the decomposition and the $L^{p}$-theory of differential forms. And in [3], we have extended the homotopy operator to the domain that is deformed to every point. In the meanwhile, the carathéodory operator $G$ form classic examples to discuss boundedness and continuity of nonlinear operators and play an important part in advanced functional analysis, and in [4] we have extended it to differential forms. In many situations, we need to estimate the various norms of the operators and their compositions.

Throughout this paper, we always assume that $\Omega$ is a bounded and convex domain and $B$ is a ball in $\mathbf{R}^{n}, n \geq 2$. Let $\sigma B$ be the ball with the same center as $B$ and with $\operatorname{diam}(\sigma B)=$ $\sigma \operatorname{diam}(B), \sigma>0$. We do not distinguish the balls from cubes in this paper. For any subset $E \subset \mathbf{R}^{n}$, we use $|E|$ to denote the Lebesgue measure of $E$. In [2], we have the estimate for $\|T(u)\|:$

$$
\|T(u)\|_{p, F} \leq 2^{n} \sigma_{n-1} \mu(\Omega)(\operatorname{diam} \Omega)\|u\|_{p, F}
$$

for all $u \in L_{l o c}^{p}\left(\Omega, \wedge^{l}\right)$, where $F \subset \Omega$ is bounded and convex. And for carathéodory operator, we obtain

$$
|f(s, \omega)| \leq a(s)+b|\omega|^{p_{1} / p_{2}}, \quad s \in \Omega, \omega \in L^{p_{1}}\left(\Omega, \wedge^{l}\right) .
$$

With these estimates, we can obtain the estimates for the composition of them. Finally, we obtain the $W^{1, p}$ norm estimates for the composition operator.

The main theorems are proved by reference to Chap. 7 of [1].

O 2012 Tang and Zhu; licensee Springer. This is an Open Access article distributed under the terms of the Creative Commons Attribution License (http://creativecommons.org/licenses/by/2.0), which permits unrestricted use, distribution, and reproduction in any medium, provided the original work is properly cited. 


\section{Some preliminaries about differential forms}

The majority of notations and preliminaries used throughout this paper can be found in [1]. For the sake of convenience, we list briefly them in this section.

Let $e_{1}, e_{2}, \ldots, e_{n}$ denote the standard orthogonal basis of $\mathbf{R}^{n}$. Suppose that $\Lambda^{l}=\Lambda^{l}\left(\mathbf{R}^{n}\right)$ is the linear space of $l$-covectors, generated by the exterior products $e_{I}=e_{i_{1}} \wedge e_{i_{2}} \wedge \cdots \wedge e_{i_{l}}$, corresponding to all ordered $l$-tuples $I=\left(i_{1}, i_{2}, \ldots, i_{l}\right), 1 \leq i_{1}<i_{2}<\cdots<i_{l} \leq n, l=0,1, \ldots, n$. The Grassmann algebra $\Lambda=\bigoplus_{l=0}^{n} \Lambda^{l}$ is a graded algebra with respect to the exterior products. For $\alpha=\Sigma \alpha^{I} e_{I} \in \Lambda$ and $\beta=\Sigma \beta^{I} e_{I} \in \Lambda$, the inner product in $\Lambda$ is given by $\langle\alpha, \beta\rangle=$ $\Sigma \alpha^{I} \beta^{I}$ with summation over all $l$-tuples $I=\left(i_{1}, i_{2}, \ldots, i_{l}\right)$ and all integrals $l=0,1, \ldots, n$. We define the Hodge star operator $\star: \Lambda \rightarrow \Lambda$ by

$$
\star \omega=\operatorname{sign}(\pi) \alpha_{i_{1}, i_{2}, \ldots, i_{k}}\left(x_{1}, x_{2}, \ldots, x_{n}\right) d x_{j_{1}} \wedge \cdots \wedge d x_{j_{n-k}},
$$

where $\omega=\alpha_{i_{1}, i_{2}, \ldots, i_{k}}\left(x_{1}, x_{2}, \ldots, x_{n}\right) d x_{i_{1}} \wedge d x_{i_{2}} \wedge \cdots \wedge d x_{i_{k}}$ is a $k$-form, $\pi=\left(i_{1}, \ldots, i_{k}, j_{1}, \ldots, j_{n-k}\right)$ is a permutation of $(1,2, \ldots, n)$ and $\operatorname{sign}(\pi)$ is the signature of the permutation. The norm of $\alpha \in \Lambda$ is given by the formula $|\alpha|^{2}=\langle\alpha, \alpha\rangle=\star(\alpha \wedge \star \alpha) \in \Lambda^{0}=\mathbf{R}$.

A differential $l$-form $\omega$ is a Schwartz distribution on $\Omega$ with values in $\Lambda^{l}\left(\mathbf{R}^{n}\right)$. We use $D^{\prime}\left(\Omega, \Lambda^{l}\right)$ to denote the space of all differential $l$-forms, and $L^{p}(\Omega, \Lambda)$ to denote the $l$-forms

$$
\omega(x)=\sum_{I} \omega(x) d x_{I}=\sum \omega_{i_{1}, i_{2}, \ldots, i_{l}}(x) d x_{i_{1}} \wedge d x_{i_{2}} \wedge \cdots \wedge d x_{i_{l}}
$$

with all coefficients $\omega_{I} \in L^{p}(\Omega, \mathbf{R})$. Thus, $L^{p}\left(\Omega, \Lambda^{l}\right), p \geq 1$, is a Banach space with norm

$$
\|\omega\|_{p}=\|\omega(x)\|_{p, \Omega}=\left(\int_{\Omega}|\omega(x)|^{p}\right)^{1 / p}=\left(\int_{\Omega}\left(\sum_{I}\left|\omega_{I}(x)\right|^{2}\right)^{p / 2} d x\right)^{1 / p} .
$$

The space $L_{1}^{p}\left(\Omega, \Lambda^{l}\right)$ is the subspace of $D^{\prime}\left(\Omega, \Lambda^{l}\right)$ with the condition

$$
\|\alpha\|_{L_{1}^{p}(\Omega)}=\left(\int_{\Omega}\left(\sum_{i=1}^{n}\left|\frac{\partial \alpha}{\partial x_{i}}\right|^{2}\right)^{p / 2} d x\right)^{1 / p}<\infty .
$$

The Sobolev space $W^{1, p}\left(\Omega, \Lambda^{l}\right)$ of $l$-forms is $W^{1, p}\left(\Omega, \Lambda^{l}\right)=L^{p}(\Omega, \Lambda) \cap L_{1}^{p}\left(\Omega, \Lambda^{l}\right)$. The norms are given by

$$
\left.\|\omega\|_{W^{1, p}(\Omega, \wedge}{ }^{l}\right)=(\operatorname{diam} \Omega)^{-1}\|\omega\|_{p, \Omega}+\|\nabla \omega\|_{p, \Omega}
$$

We denote the exterior derivative by $d: D^{\prime}\left(\Omega, \Lambda^{l}\right) \rightarrow D^{\prime}\left(\Omega, \Lambda^{l+1}\right)$ for $l=0,1, \ldots, n-1$, which means

$$
d \omega(x)=\sum_{k=1}^{n} \sum_{1 \leq i_{1}<\cdots<i_{l} \leq n} \frac{\partial \omega_{i_{1}, i_{2}, \ldots, i_{l}}(x)}{\partial x_{k}} d x_{k} \wedge d x_{i_{1}} \wedge d x_{i_{2}} \wedge \cdots \wedge d x_{i_{l}}
$$

Its formal adjoint operator is defined by

$$
d^{*}=(-1)^{n l+1} \star d \star: D^{\prime}\left(\Omega, \Lambda^{l+1}\right) \rightarrow D^{\prime}\left(\Omega, \Lambda^{l}\right), \quad l=0,1, \ldots, n-1,
$$

which is called the Hodge codifferential. 
In [3], we define an operation $K_{y}$ for any $y \in \Omega$ and we construct a homotopy operator $T: C^{\infty}\left(\Omega, \wedge^{l}\right) \rightarrow C^{\infty}\left(\Omega, \wedge^{l}\right)$ by averaging $K_{y}$ over all points $y \in \Omega$ :

$$
T \omega=\int_{\Omega} \psi(y) K_{y}\left(\phi^{*} \omega\right) d y
$$

where $\psi$ in $C_{0}^{\infty}(U)$ is normalized so that $\int_{\Omega} \psi(y) d y=1$. We obtain the following decomposition for the operator $T$ :

$$
\omega=d(T \omega)+T(d \omega)
$$

From [2], we know that for any differential form $u \in L_{l o c}^{p}\left(\Omega, \wedge^{l}\right), l=1,2, \ldots, n, 1<p<\infty$, we have

$$
\begin{aligned}
& \|\nabla(T u)\|_{p, \Omega} \leq C \mu(\Omega)\|u\|_{p, \Omega}, \\
& \|T u\|_{p, \Omega} \leq C \mu(\Omega) \operatorname{diam}(B)\|u\|_{p, \Omega} .
\end{aligned}
$$

where $\mu(B)$ is flatness of $\Omega$ (see [2]). See [5-13] for more details of differential forms and its applications.

Then we define the carathéodory conditions and carathéodory operator for differential forms (see [4]).

Definition 2.1 For a mapping $f: \Omega \times \wedge^{l} \rightarrow \wedge^{l}$, where $\Omega$ is an open set in $\mathbf{R}^{n}$, we say that $f$ satisfies carathéodory conditions if

1. For all most $s \in \Omega, f(s, \omega)$ is continuous with respect to $\omega$, which means that $f$ can be expanded as $f(s, \omega)=\sum_{J} f_{J}(s, \omega) d x_{J}$, where $f_{J}: \Omega \times \wedge^{l} \rightarrow \mathbf{R}$ and $f_{J}(s, \omega)$ is continuous about $\omega$ for all most $s \in \Omega$; and

2. For any fixed $\omega=\sum_{I} \omega d x_{I} \in \wedge^{l}, f(s, \omega)$ is measurable about $s$, which means that each coefficient function $f_{J}(s, \omega)$ is measurable about $s$ for any fixed $\omega \in \wedge^{l}$.

Throughout this paper, we assume that $f(s, \omega)$ satisfies the carathéodory condition $(C$ condition). Similarly, we can define the continuity of $f(s, \omega)$ about $(s, \omega) \in \Omega \times \wedge^{l}$.

Definition 2.2 Suppose that $\Omega \subset \mathbf{R}^{n}$ is a measurable set $(0<\operatorname{mes} \Omega \leq+\infty)$, and $f: \Omega \times$ $\wedge^{l} \rightarrow \wedge^{l}$. We define the carathéodory operator $G: \wedge^{l} \rightarrow \wedge^{l}$ for differential forms by

$$
G \omega(s)=f(s, \omega(s))
$$

For the carathéodory operator, we have the similar result for differential forms as for the functions (see [4]).

Theorem 2.1 The carathéodory operator $G$ maps continuously and boundedly $L^{p_{1}}\left(\Omega, \wedge^{l}\right)$ into $L^{p_{2}}\left(\Omega, \wedge^{l}\right)$, if and only if, there exists $b>0, a(x) \geq 0, a(x) \in L^{p_{2}}(\Omega)$ satisfying the following inequality:

$$
|f(x, \omega)| \leq a(x)+b|\omega|^{\frac{p_{1}}{p_{2}}} \quad\left(x \in \Omega, \omega \in \wedge^{l}\right) .
$$

Here, we suppose $p_{1}=p_{2}$. 
We define Muckenhoupt weights (see [1]).

Definition 2.3 A weight $\omega$ satisfies the $A_{r}(\Omega)$-condition in a subset $\Omega \subset \mathbf{R}^{n}$, where $r>1$, and write $\omega \in A_{r}(\Omega)$ when

$$
\sup _{B}\left(\frac{1}{|B|} \int_{B} \omega d x\right)\left(\frac{1}{|B|} \int_{B} \omega^{1 /(1-r)} d x\right)^{r-1}<\infty
$$

where the supremum is over all balls $B \subset \Omega$.

The following class of two-weight or $A_{r, \lambda}(\Omega)$-weights appeared in [1] and [13].

Definition 2.4 A pair of weights $\left(\omega_{1}, \omega_{2}\right)$ satisfy the $A_{r, \lambda}(\Omega)$-condition in a set $B \subset R^{N}$, write $\left(\omega_{1}, \omega_{2}\right) \in A_{r, \lambda}(B)$ for some $\lambda \geq 1$ and $1<r<\infty$ with $1 / r+1 / r^{\prime}=1$, if

$$
\sup _{B \subset \Omega}\left(\frac{1}{|B|} \int_{B} \omega_{1}^{\lambda} d x\right)^{1 / \lambda r}\left(\frac{1}{|B|} \int_{B}\left(1 / \omega_{2}\right)^{1 /(r-1)} d x\right)^{\lambda(r-1)}<\infty
$$

for all balls $B \subset \Omega$.

In the present paper, we deal with the A-harmonic equations formulated by $d^{*} A(x, d u)=$ $B(x, d u)$.

We also need the following weak reverse Hölder inequality (Lemma 3.1.1 of [1]).

Theorem 2.2 Let $u$ be a solution of the nonhomogeneous A-harmonic equation in a domain $\Omega$ and $0<s, t<\infty$. Then there exists a constant $C$, independent of $u$, such that

$$
\|u\|_{s, B} \leq C|B|^{(t-s) / s t}\|u\|_{t, \rho B}
$$

for all balls $B$ with $\rho B \subset \Omega$ for some $\rho>1$.

For $A_{r}$-weights $\omega$, we have the following reverse Hölder inequality (Lemma 1.4.7 of [1]).

Theorem 2.3 If $\omega \in A_{r}, r>1$, then there exist constants $\beta>1$ and $C$, independent of $\omega$, such that

$$
\|\omega\|_{\beta, Q} \leq C|Q|^{(1-\beta) / \beta}\|\omega\|_{1, Q}
$$

for all balls $Q \subset \mathbf{R}^{N}$.

\section{Main results and proofs}

Theorem 3.1 Let $u \in L_{l o c}^{s}\left(\Omega, \wedge^{l}\right), l=1,2, \ldots, n, 1<s<\infty$, be a solution of the A-harmonic equation in domain $\Omega$ is bounded and convex and $T: C^{\infty}\left(\Omega, \wedge^{l}\right) \rightarrow C^{\infty}\left(\Omega, \wedge^{l-1}\right)$ be the homotopy operator. Assume that $\rho>1$ and $\omega \in A_{r}(\Omega)$ for some $1<r<\infty$. Then $T(G(u)) \in$ $L_{\text {loc }}^{s}\left(\Omega, \wedge^{l}\right)$. Moreover, there exists a constant $C$, independent of $u$, such that

$$
\|T(G(u))\|_{s, B, \omega^{\alpha}} \leq C|B| \operatorname{diam}(B)\|u\|_{s, \rho B, \omega^{\alpha}}
$$

for all balls $B$ with $\rho B \subset \Omega$ and any real number $\alpha$ with $0<\alpha \leq 1$. 
Proof We only need to prove the inequality holds. With (2.6) and (2.7), we have

$$
\begin{aligned}
\|T(G(u))\|_{s, B} & \leq C \mu(\Omega) \operatorname{diam} B\|G(u)\|_{s, B} \\
& \leq C \mu(\Omega) \operatorname{diam} B\|a(x)+b|u|\|_{s, B} \\
& \leq C \mu(\Omega) \operatorname{diam} B\left(\|a(x)\|_{s, B}+b\|u\|_{s, B}\right) \\
& \leq C_{1} \mu(\Omega) \operatorname{diam} B\|u\|_{s, B} .
\end{aligned}
$$

Then just like the process of the proof for Theorem 7.3.14 in [1], we obtain the inequality.

We discuss the inequality with $0<\alpha<1$ and $\alpha=1$ separately. For $0<\alpha<1$, first we set $t=s /(1-\alpha)$. With Hölder inequality, we obtain

$$
\begin{aligned}
\|T(G(u))\|_{s, B, \omega^{\alpha}} & =\left(\int_{B}\left((|T(G(u))|) \omega^{\alpha / s}\right)^{s} d x\right)^{1 / s} \\
& \leq\|T(G(u))\|_{t, B}\left(\int_{B} \omega^{t \alpha /(t-s)} d x\right)^{(t-s) / s t} \\
& =\|T(G(u))\|_{t, B}\left(\int_{B} \omega d x\right)^{\alpha / s} .
\end{aligned}
$$

By (3.2), we obtain

$$
\|T(G(u))\|_{t, B} \leq C_{3} \mu(\Omega) \operatorname{diam} B\|u\|_{t, B} .
$$

Let $m=s /(1+\alpha(r-1))$, then $m<s$. With (3.3) and (3.4) and using Theorem 2.2, we have

$$
\begin{aligned}
\|T(G(u))\|_{s, B, \omega^{\alpha}} & \leq C_{3} \mu(\Omega) \operatorname{diam} B\|u\|_{t, B}\left(\int_{B} \omega d x\right)^{\alpha / s} \\
& \leq C_{4} \mu(\Omega) \operatorname{diam} B|B|^{(m-t) / m t}\|u\|_{m, \rho B}\left(\int_{B} \omega d x\right)^{\alpha / s} .
\end{aligned}
$$

And using Hölder's inequality again, we obtain

$$
\begin{aligned}
\|u\|_{m, \rho B} & =\left(\int_{\rho B}|u|^{m} d x\right)^{1 / m} \\
& =\left(\int_{\rho B}\left(|u| \omega^{\alpha / s} \omega^{-\alpha / s}\right)^{m} d x\right)^{1 / m} \\
& \leq\left(\int_{\rho B}|u|^{s} \omega^{\alpha} d x\right)^{1 / s}\left(\int_{\rho B}(1 / \omega)^{1 /(r-i)} d x\right)^{\alpha(r-1) / s}
\end{aligned}
$$

for all balls $B$ with $\rho B \subset \Omega$. With (3.5) and (3.6), we find that

$$
\begin{aligned}
\|T(G(u))\|_{s, B, \omega^{\alpha}} \leq & C_{4} \mu(\Omega) \operatorname{diam} B|B|^{(m-t) / m t}\|u\|_{s, \rho B, \omega^{\alpha}} \\
& \times\left(\int_{\rho B}(1 / \omega)^{1 /(r-1)} d x\right)^{\alpha(r-1) / s}\left(\int_{B} \omega d x\right)^{\alpha / s} .
\end{aligned}
$$


As $\omega \in A_{r}(\Omega)$, we have

$$
\begin{aligned}
& \left(\int_{\rho B}(1 / \omega)^{1 /(r-1)} d x\right)^{\alpha(r-1) / s}\left(\int_{B} \omega d x\right)^{\alpha / s} \\
& \quad \leq\left(\left(\int_{\rho B} \omega d x\right)\left(\int_{\rho B}(1 / \omega)^{1 /(r-1)} d x\right)^{r-1}\right)^{\alpha / s} \\
& \quad=\left(|\rho B|^{r}\left(\frac{1}{|\rho B|} \int_{\rho B} \omega d x\right)\left(\frac{1}{|\rho B|} \int_{\rho B}(1 / \omega)^{1 /(r-1)} d x\right)^{r-1}\right)^{\alpha / s} \\
& \leq C_{5}|B|^{\alpha r / s} .
\end{aligned}
$$

With (3.7) and (3.8), we have

$$
\|T(G(u))\|_{s, B, \omega^{\alpha}} \leq C_{6} \mu(B) \operatorname{diam} B\|u\|_{s, \rho B, \omega^{\alpha}}
$$

for all balls $B$ with $\rho B \subset \Omega$. This is just (3.1) with $0<\alpha<1$. Then we prove the case of $\alpha=1$. First, with Theorem 2.3, we know

$$
\|\omega\|_{\beta, B} \leq C_{7}|B|^{(1-\beta) / \beta}\|\omega\|_{1, B}
$$

here $\beta>1$ and $C_{7}>0$ are all constants. Let $t=s \beta /(\beta-1)$, then we know $1<s<t$ and $\beta=t /(t-s)$. With Hölder's inequality (3.2), and (3.10), we obtain

$$
\begin{aligned}
\|T(G(u))\|_{s, B, \omega} & =\left(\int_{B}\left(|T(G(u))| \omega^{1 / s}\right)^{s} d x\right)^{1 / s} \\
& \left.\leq\left(\int_{B}|T(G(u))|^{t} d x\right)^{1 / t}\left(\int_{B}\left(\omega^{1 / s}\right)^{s t /(t-s)} d x\right)^{(t-s) / s t}\right) \\
& =C_{8}\|T(G(u))\|_{t, B}\|\omega\|_{\beta, B}^{1 / s} \\
& \leq C_{8} \mu(B) \operatorname{diam} B\|u\|_{t, B}\|\omega\|_{\beta, B}^{1 / s} \\
& \leq C_{9} \mu(B) \operatorname{diam} B|B|^{(1-\beta) / \beta s}\|\omega\|_{\beta, B}^{1 / s}\|u\|_{t, B} \\
& \leq C_{9} \mu(B) \operatorname{diam} B|B|^{-1 / t}\|\omega\|_{\beta, B}^{1 / s}\|u\|_{t, B} .
\end{aligned}
$$

Set $m=s / r$. With Theorem 2.2, we have

$$
\|u\|_{t, B} \leq C_{10}|B|^{(m-t) / m t}\|u\|_{m, \rho B}
$$

And we use Hölder's inequality again

$$
\begin{aligned}
\|u\|_{m, \rho B} & =\left(\int_{\rho B}\left(|u| \omega^{1 / s} \omega^{-1 / s}\right)^{m} d x\right)^{1 / m} \\
& \leq\left(\int_{\rho B}|u|^{s} \omega d x\right)^{1 / s}\left(\int_{\rho B}(1 / \omega)^{1 /(r-1)} d x\right)^{(r-1) / s} .
\end{aligned}
$$

With $\omega \in A_{r}(\Omega)$, we have

$$
\|\omega\|_{1, B}^{1 / s}\|1 / \omega\|_{1 /(r-1), \rho B}^{1 / s} \leq\left(\left(\int_{\rho B} \omega d x\right)\left(\int_{\rho B}(1 / \omega)^{1 /(r-1)} d x\right)^{r-1}\right)^{1 / s}
$$




$$
\begin{aligned}
& =\left(|\rho B|^{r}\left(\frac{1}{|\rho B|} \int_{\rho B} \omega d x\right)\left(\frac{1}{|\rho B|} \int_{\rho B}(1 / \omega)^{1 /(r-1)} d x\right)^{r-1}\right)^{1 / s} \\
& \leq C_{11}|B|^{r / s} .
\end{aligned}
$$

With (3.11)-(3.14), we have

$$
\begin{aligned}
\|T(G(u))\|_{s, B, \omega} & \leq C_{12} \mu(B) \operatorname{diam} B|B|^{-1 / t}\|\omega\|_{1, B}^{1 / s}|B|^{(m-t) / m t}\|u\|_{m, \rho B} \\
& \leq C_{12} \mu(B) \operatorname{diam} B|B|^{-1 / m}\|\omega\|_{1, B}^{1 / s}\|1 / \omega\|_{1 /(r-1), \rho B}^{1 / s}\|u\|_{s, \rho B, \omega} \\
& \leq C_{13} \mu(B) \operatorname{diam} B\|u\|_{s, \rho B, \omega}
\end{aligned}
$$

for all balls $B$ with $\rho B \subset \Omega$. Thus, we complete the proof.

Actually by the method developed in $[1]$, for the two weight $\left(\omega_{1}, \omega_{2}\right) \in A_{r, \lambda}(\Omega)$, we have the following inequality.

Theorem 3.2 Let $u \in L_{l o c}^{s}\left(\Omega, \wedge^{l}\right), l=1,2, \ldots, n, 1<s<\infty$, be a solution of the A-harmonic equation in domain $\Omega$ is bounded and convex and $T: C^{\infty}\left(\Omega, \wedge^{l}\right) \rightarrow C^{\infty}\left(\Omega, \wedge^{l-1}\right)$ be the homotopy operator. Assume that $\rho>1$ and $\left(\omega_{1}, \omega_{2}\right) \in A_{r, \lambda}(\Omega)$ for some $1<r<\infty, \lambda \geq 1$. Then, $T(G(u)) \in L_{\text {loc }}^{S}\left(\Omega, \wedge^{l}\right)$. Moreover, there exists a constant $C$, independent of $u$, such that

$$
\|T(G(u))\|_{s, B, \omega_{1}^{\alpha}} \leq C|B| \operatorname{diam}(B)\|u\|_{s, \rho B, \omega_{2}^{\alpha}}
$$

for all balls $B$ with $\rho B \subset \Omega$ and any real number $\alpha$ with $0<\alpha \leq 1$.

Proof Let $t=\lambda s /(\lambda-\alpha)$. As $\frac{1}{s}=\frac{1}{t}+\frac{(t-s)}{s t}$, with Hölder inequality, we have

$$
\begin{aligned}
\left(\int_{B}|T(G u)| \omega_{1}^{\alpha} d x\right)^{1 / s} & =\left(\int_{B}\left(|T(G u)| \omega_{1}^{\alpha / s}\right)^{s} d x\right)^{1 / s} \\
& \leq\left(\int_{B}|T(G u)|^{t} d x\right)^{1 / t}\left(\int_{B}\left(\omega_{1}^{\alpha / s}\right)^{s t /(t-s)} d x\right)^{(t-s) / s t} \\
& \leq\|T(G u)\|_{t, B}\left(\int_{B} \omega_{1}^{\lambda} d x\right)^{\alpha / \lambda s}
\end{aligned}
$$

for all balls $B \subset \Omega$. Then, from (3.2), we obtain

$$
\|T(G u)\|_{t, B} \leq C_{1} \mu(\Omega) \operatorname{diam} B\|u\|_{t, B} .
$$

Let $m=\frac{\lambda s}{(\lambda+\alpha(r-1))}$, then we know $m<s<t$. With (3.17) and (3.18) and Theorem 2.2, we have

$$
\begin{aligned}
& \left(\int_{B}|T(G u)|^{s} \omega_{1}^{\alpha} d x\right)^{1 / s} \\
& \quad \leq C_{1}|B| \mu(\Omega) \operatorname{diam} B\|u\|_{t, B}\left(\int_{B} \omega_{1}^{\lambda} d x\right)^{\alpha / \lambda s} \\
& \quad \leq C_{2}|B||B|^{\frac{m-t}{m t}}\|u\|_{m, \rho B}\left(\int_{B} \omega_{1}^{\lambda} d x\right)^{\alpha / \lambda s}
\end{aligned}
$$


Then by the generalized Hölder's inequality, we have

$$
\begin{aligned}
\|u\|_{m, \rho B} & =\left(\int_{\rho B}|u|^{m} d x\right)^{1 / m} \\
& =\left(\int_{\rho B}\left(|u| \omega_{2}^{\alpha / s} \omega_{2}^{-\alpha / s}\right)^{m} d x\right)^{1 / m} \\
& \leq\left(\int_{\rho B}|u|^{s} \omega_{2}^{\alpha} d x\right)^{1 / s}\left(\int_{\rho B}\left(1 / \omega_{2}\right)^{\lambda /(r-1)} d x\right)^{\alpha(r-1) / \lambda s}
\end{aligned}
$$

for all balls $B$ with $\rho B \subset \Omega$, where we use $\frac{1}{m}=\frac{1}{s}+\frac{s-m}{s m}$. Then with (3.19) and (3.20), we obtain

$$
\begin{aligned}
& \left(\int_{B}|T(G u)|^{s} \omega_{1}^{\alpha} d x\right)^{1 / s} \\
& \leq C_{3}|B||B|^{\frac{m-t}{m t}} \mu(\Omega) \operatorname{diam} B\left(\int_{\rho B}|u|^{s} \omega_{2}^{\alpha} d x\right)^{1 / s} \\
& \quad \times\left(\int_{\rho B}\left(1 / \omega_{2}\right)^{\lambda /(r-1)} d x\right)^{\frac{\alpha(r-1)}{\lambda s}}\left(\int_{B} \omega_{1}^{\lambda} d x\right)^{\alpha / \lambda s} .
\end{aligned}
$$

Then, with $\left(\omega_{1}, \omega_{2}\right) \in A_{r, \lambda}(\Omega)$, we have

$$
\begin{aligned}
& \left(\left(\int_{B} \omega_{1}^{\lambda} d x\right)^{\alpha / \lambda s}\left(\int_{\rho B}\left(1 / \omega_{2}\right)^{\lambda /(r-1)} d x\right)^{r-1}\right)^{\alpha / \lambda s} \\
& \leq\left(\left(\int_{\rho B} \omega_{1}^{\lambda} d x\right)^{\alpha / \lambda s}\left(\int_{\rho B}\left(1 / \omega_{2}\right)^{\lambda /(r-1)} d x\right)^{r-1}\right)^{\alpha / \lambda s} \\
& =|\rho B|^{r}\left(\frac{1}{|\rho B|} \int_{\rho B} \omega_{1}^{\lambda} d x\right)\left(\frac{1}{|\rho B|} \int_{\rho B}\left(\frac{1}{\omega_{2}^{\lambda}}\right)^{1 /(r-1)}\right)^{\alpha / \lambda s} \\
& \leq C_{4}|B|^{\alpha r / \lambda s} .
\end{aligned}
$$

With (3.21) and (3.22), we have

$$
\left(\int_{B}|T(G u)|^{s} \omega_{1}^{\alpha} d x\right)^{1 / s} \leq C \mu(\Omega) \operatorname{diam} B\left(\int_{\rho B}|u|^{s} \omega_{2}^{\alpha} d x\right)^{1 / s}
$$

for all balls $B$ with $\rho B \subset \Omega$.

For the compositions of the gradient operator $\nabla$, the homotopy operator $T$, the carathéodory operator $G, \nabla \circ T \circ G$, we obtain the local Sobolev-Poincaré embedding theorem.

Theorem 3.3 Let $u \in L_{l o c}^{s}\left(\Omega, \wedge^{l}\right), l=1,2, \ldots, n, 1<s<\infty$, be a solution of the A-harmonic equation in bounded and convex domain $\Omega, T: C^{\infty}\left(\Omega, \wedge^{l}\right) \rightarrow C^{\infty}\left(\Omega, \wedge^{l-1}\right)$ be the homotopy operator, $\nabla$ be the gradient operator and $G$ be the carathéodory operator. Then $\nabla(T(G(u))) \in L_{\text {loc }}^{s}\left(\Omega, \wedge^{l}\right)$ and $T(G(u)) \in W^{1, s}(B)$. Moreover, there exists a constant $C$, inde- 
pendent of $u$, such that

$$
\|\nabla(T(G(u)))\|_{s, B} \leq C \mu(\Omega)\|u\|_{s, B}
$$

and

$$
\|T(G(u))\|_{W^{1, S(B)}} \leq C \mu(\Omega)\|u\|_{s, B} .
$$

Proof Actually, we only need to prove (3.16) and (3.17). From these two inequalities, the remaining part of the theorem follows. From (2.4), we obtain

$$
\|\nabla(T(\omega))\|_{p, \Omega} \leq C \mu(\Omega)\|\omega\|_{p, \Omega}
$$

for any $\omega \in L_{l o c}^{p}\left(\wedge^{l} B\right)$. Let $G(u)=\omega$, we have

$$
\begin{aligned}
\|\nabla(T(G(u)))\|_{s, B} & \leq C \mu(\Omega)\|G(u)\|_{s, B} \\
& \leq C \mu(\Omega)\left(\|a(x)\|_{s, B}+b\|u\|_{s, B}\right) \\
& \leq C_{1} \mu(\Omega)\|u\|_{s, B} .
\end{aligned}
$$

With the definition of $W^{1, p}$ norm, (3.2), and (3.27), we have

$$
\begin{aligned}
\|T(G(u))\|_{W^{1, p}(B)} & =\operatorname{diam}(B)^{-1}\|T(G(u))\|_{s, B}+\|\nabla(T(G(u)))\|_{s, B} \\
& \leq \operatorname{diam}(B)^{-1} C_{1} \mu(\Omega) \operatorname{diam} B\|u\|_{s, B}+C \mu(\Omega)\|u\|_{s, B} \\
& \leq C_{2} \mu(\Omega)\|u\|_{s, B} .
\end{aligned}
$$

Thus, we obtain the inequality.

Using the same method as in the proof of Theorem 3.1, we obtain the weighted inequality for $\|\nabla(T(G(u)))\|_{s, B, \omega^{\alpha}}$.

Corollary 3.4 Let $u \in L_{\text {loc }}^{s}\left(\Omega, \wedge^{l}\right), l=1,2, \ldots, n, 1<s<\infty$, be a solution of the A-harmonic equation in bounded and convex domain $\Omega, T: C^{\infty}\left(\Omega, \wedge^{l}\right) \rightarrow C^{\infty}\left(\Omega, \wedge^{l-1}\right)$ be the homotopy operator, $\nabla$ be the gradient operator and $G$ be the carathéodory operator. Assume that $\rho>1$ and $\omega \in A_{r}(\Omega)$ for some $1<r<\infty$. Then $\nabla(T(G(u))) \in L_{\text {loc }}^{s}\left(\Omega, \wedge^{l}\right)$. Moreover, there exists a constant $C$, independent of $u$, such that

$$
\|\nabla(T(G(u)))\|_{s, B, \omega^{\alpha}} \leq C \mu(\Omega)\|u\|_{s, B, \omega^{\alpha}} .
$$

For $G(T(u))$, we also have the similar result.

Corollary 3.5 Let $u \in L_{\text {loc }}^{s}\left(\Omega, \wedge^{l}\right), l=1,2, \ldots, n, 1<s<\infty$, be a solution of the A-harmonic equation in a bounded, convex domain $\Omega$ and $T: C^{\infty}\left(\Omega, \wedge^{l}\right) \rightarrow C^{\infty}\left(\Omega, \wedge^{l-1}\right)$ be the homotopy operator. Assume that $\rho>1$ and $\omega \in A_{r}(\Omega)$ for some $1<r<\infty$. Then $G(T(u)) \in$ $L_{\text {loc }}^{s}\left(\Omega, \wedge^{l}\right)$. Moreover, there exists a constant $C$, independent of $u$, such that

$$
\|G(T(u))\|_{s, B, \omega^{\alpha}} \leq C|B| \operatorname{diam}(B)\|u\|_{s, \rho B, \omega^{\alpha}}
$$

for all balls $B$ with $\rho B \subset \Omega$ and any real number $\alpha$ with $0<\alpha \leq 1$. 
Proof If (3.22) holds, then $G(T(u)) \in L_{l o c}^{s}\left(\Omega, \wedge^{l}\right)$ follows. Hence, we only need to prove (3.22). From (2.6) and (2.7), we have

$$
\begin{aligned}
\|G(T(u))\|_{s, B} & \leq\|a(x)\|_{s, B}+b\|T(u)\|_{s, B} \\
& \leq C \mu(\Omega) \operatorname{diam}(B)\|u\|_{s, B} .
\end{aligned}
$$

Using the method in the proof of Theorem 3.1, we obtain the inequality.

Actually for two weight $\left(\omega_{1}, \omega_{2}\right) \in A_{r, \lambda}(\Omega)$, for some $\lambda \leq 1$ and $1<r<\infty$, we have the similar inequalities, with the method developed in the proof of Theorem 3.2.

Corollary 3.6 Let $u \in L_{l o c}^{s}\left(\Omega, \wedge^{l}\right), l=1,2, \ldots, n, 1<s<\infty$, be a differential form satisfying A-harmonic equation in a bounded, convex domain $\Omega \subset R^{N}$ and $T: C^{\infty}\left(\Omega, \wedge^{l}\right) \rightarrow$ $C^{\infty}\left(\Omega, \wedge^{l-1}\right)$ be the homotopy operator defined in (2.2). Assume $\rho>1$ and $\left(\omega_{1}, \omega_{2}\right) \in A_{r, \lambda}(\Omega)$ for some $\lambda \geq 1$ and $1<r<\infty$. Then there exists a constant $C$, independent of $u$, such that

$$
\left(\int_{B}|\nabla(T(u))|^{s} \omega_{1}^{\alpha} d x\right)^{1 / s} \leq C|B|\left(\int_{\rho B}|u|^{s} \omega_{2}^{\alpha} d x\right)^{1 / s}
$$

for all balls $B$ with $\rho B \subset \Omega$ and all real number $\alpha$ with $0<\alpha<\lambda$.

The above inequality is an extension of the usual inequality of $A_{r}$-weights. If we choose $\omega_{1}(x)=\omega_{2}(x)=\omega(x)$ and $\lambda=1$ in the two weighted inequalities, we obtain the $A_{r}(\Omega)$ weight case.

\section{Competing interests}

The authors declare that they have no competing interests.

\section{Authors' contributions}

Professor JZ gave the ideas. ZT gave the proofs and completed the manuscript.

\section{Acknowledgement}

The research of the first author was supported by the NSF of Hunan Province (No. 11JJ3004) and NSF of NUDT (No. JC10-02-02).

Received: 18 October 2011 Accepted: 6 June 2012 Published: 31 August 2012

\section{References}

1. Agarwal, PR, Ding, S, Nolder, CA: Inequalities for Differential Forms. Springer, New York (2009)

2. Iwaniec, T, Lutoborski, A: Integral estimates for null Lagrangians. Arch. Ration. Mech. Anal. 125, 25-79 (1993)

3. Tang, Z, Zhu, J, Huang, J, Li, J: An extension of the Poincaré lemma of differential forms. In: World Congress on Engineering and Technology, vol. 2, pp. 403-406. IEEE, New York (2011)

4. Tang, Z, Zhu, J: Carathéodory operator of differential forms. J. Inequal. Appl. 2011, 88 (2011). doi:10.1186/1029-242X-2011-88

5. Zhu, J, Li, J: Some priori estimates about solutions to nonhomogeneous A-harmonic equations. J. Inequal. Appl. 2010, Article ID 520240 (2010)

6. Ding, S: Two-weight Caccioppoli inequalities for solutions of nonhomogeneous A-harmonic equations on Riemannian manifolds. Proc. Am. Math. Soc. 132, 2367-2375 (2004)

7. Ding, S: Local and global norm comparison theorems for solutions to the nonhomogeneous A-harmonic equation. J. Math. Anal. Appl. 335, 1274-1293 (2007)

8. Ding, S, Zhu, J: Poincaré-type inequalities for the homotopy operator with $L_{\varphi}(\Omega)$-norms. Nonlinear Anal., Theory Methods Appl. 74(11), 3728-3735 (2011)

9. Nolder, CA: Hardy-Littlewood theorems for A-harmonic tensors. III. J. Math. 43, 613-631 (1999)

10. Nolder, CA: Global integrability theorems for A-harmonic tensors. J. Math. Anal. Appl. 247, 236-247 (2000)

11. Nolder, CA: Conjugate harmonic functions and Clifford algebras. J. Math. Anal. Appl. 302, 137-142 (2005)

12. Serrin, J: Local behavior of solutions of quasi-linear equations. Acta Math. 111, 247-302 (1964)

13. Neugebauer, CJ: Inserting $A_{p}$-weights. Proc. Am. Math. Soc. 87, 644-648 (1983)

14. Zhu, J, Ding, S, Tang, Z: The reverse Hölder and Caccioppoli type inequalities for generalized A-harmonic equations (in press) 
doi:10.1186/1029-242X-2012-188

Cite this article as: Tang and Zhu: Estimates for the composition of the carathéodory and homotopy operators. Journal of Inequalities and Applications 2012 2012:188.

Submit your manuscript to a SpringerOpen ${ }^{\circ}$ journal and benefit from:

- Convenient online submission

- Rigorous peer review

- Immediate publication on acceptance

Open access: articles freely available online

- High visibility within the field

- Retaining the copyright to your article

Submit your next manuscript at $\gg$ springeropen.com 